\title{
A Spinning Disk as a Spatial Light Modulator for Rapid Infrared Imaging
}

\author{
Zijian Zhang ${ }^{1}$, Lin Liu ${ }^{1}$, Aznida Abu Bakar Sajak ${ }^{1}$, Lu Gan $^{2}$, Yi Huang ${ }^{1}$, Yaochun Shen ${ }^{1}$ * \\ ${ }^{1}$ Department of Electrical Engineering and Electronics, University of Liverpool, Liverpool, \\ United Kingdom \\ ${ }^{2}$ Electronic and Computer Engineering, Brunel University, London, United Kingdom \\ ycshen@liverpool.ac.uk
}

\begin{abstract}
This paper reports a novel spinning disk approach, to achieve video-rate compressive imaging at infrared (IR) and terahertz (THz) frequencies. It uses a 200-mm-diameter circular stainless steel disk with a number of chemically etched 2-mm-diameter holes as a spatial light modulator (SLM). We demonstrate that this mechanical SLM is well suited for imaging at both optical and THz frequencies since the stainless steel is opaque to all optical and electromagnetic radiations whilst the holes are totally transparent. Using a single pair of IR emitter and receiver, we demonstrate that the system is capable of capturing a 96×96 video sequence at 10 frames/second. The achieved spatial resolution is better than 2 $\mathrm{mm}$ using the spinning disk where the diameter of the holes is $2 \mathrm{~mm}$. We also present a key-frame extraction method based on this SLM disk, which allows us to capture the shape of a sample even when its size is larger than the effective imaging area of the system.
\end{abstract}

\section{Introduction}

Compressed sensing (CS) provides a novel signal sampling and reconstruction technique, where sparse signals can be recovered from a small number of measurements [1 2]. Such an approach offers a promising solution for imaging in the infrared (IR) or terahertz $(\mathrm{THz})$ spectral regions as only a single detector, rather than a sensor array is required [3]. In addition, CS imaging can dramatically decreases the measurement time as compared with the conventional raster scanning method, since fewer signal samples are required to reconstruct an image [4].

Let $\boldsymbol{x}$ represent the vector version of an image with $N$ pixels. Suppose that $\boldsymbol{x}$ has a $K$-sparse representation $\widehat{\boldsymbol{x}}$ under a sparsity basis $\boldsymbol{\Psi}$ (e.g. the discrete cosine transform or the wavelet). The CS sampling process can be described as $[1,2]$

$$
y=\Phi x=
$$

\section{$\Phi \Psi \widehat{x}$}

where $\boldsymbol{y}$ is the $M \times 1$ measured signal vector, $\boldsymbol{\Phi}$ is an $M \times N(M \ll N)$ measurement vector. The original signal $\boldsymbol{x}$ then can be obtained by solving an optimization problem of $\ell_{0}$ norm:

$$
\min _{\widehat{x}}\|\widehat{x}\|_{0} \text { s.t. } A \widehat{x}=
$$

$y$

satisfying 


$$
y=\Phi \Psi \widehat{x}
$$

where $\boldsymbol{A}=\boldsymbol{\Phi} \boldsymbol{\Psi}$. In addition, if $\boldsymbol{A}$ satisfies the restricted isometry property (RIP) [1, 2], one can reconstruct $\boldsymbol{x}$ reliably. It is known that the RIP holds if the measurement matrix $\boldsymbol{\Phi}$ is incoherent with the sparsity basis $\boldsymbol{\Psi}$ [5 6]. Typical choices of measurement matrices include the Gaussian random matrix, the Bernoulli matrix, Toeplitz matrix, randomized partial Hadamard matrix and so on [7-9].

In a practical compressive imaging system, the measurement matrix is implemented using a spatial light modulator (SLM) to vary the amplitude of light waves based on mechanical or electro-optical methods. Practical applications find that the mechanical one is well suited for imaging at both optical and $\mathrm{THz}$ frequencies [10-13]. In our previous work, a single pixel THz imaging idea was developed [12]. We introduced a spinning disk with a number of 1-mm-diameter square holes designed from a nearly block Toeplitz matrix as an SLM. However, the imaging performance of this system is limited since the scattering effect of $\mathrm{THz}$ radiation at the sharp corners will degrade the overall signal to noise rate.

In this paper, we present further computer simulations and hardware implementations of a novel spinning disk approach which is aimed at achieving video rate compressed IR imaging. By utilizing our new generation of disk with a set of randomly positioned $2 \mathrm{~mm}$ circular holes, we achieved a spatial resolution of better than $2 \mathrm{~mm}$. In addition, the good quality IR images with frame rate as fast as 10 frames per second are captured and reconstructed. The image acquisition rate is limited only by the speed of the electric motor used in our experiment. Furthermore, a key-frame extraction method based on the SLM disk was proposed. It enables the sample with a size larger than the field of view to be extracted from the captured video.

\section{Method}

\subsection{Novel SLM Spinning Disk Design}

Fig. 1 shows the schematic diagram of our novel SLM spinning disk. This disk is made of a 0.28 -mm-thick stainless plate with a diameter of $200 \mathrm{~mm}$. A number of randomly positioned circular holes with 2-mm-diameter are chemically etched on the disk. Each hole is $100 \%$ transparent to the IR light while the stainless steel is $100 \%$ opaque to the light. The square enclosed by the red line in Fig. 1 is the zoom-in window that represents one of effective imaging areas with a size of $32 \mathrm{~mm} \times 32 \mathrm{~mm}$.

In previous studies [12,13], printed circuit boards (PCBs) have been used as an SLM. The PCB substrate material is mostly opaque to IR radiation and it also absorbs strongly $\mathrm{THz}$ radiations particularly at higher frequencies. In addition, the absorption and the dispersion caused by the PCB substrate may not 
be uniform across the whole substrate and this will affect the encoding accuracy thus weakening the quality of the reconstructed images. More importantly, the $\mathrm{THz}$ radiation spans a frequency range from $300 \mathrm{GHz}$ to $10 \mathrm{THz}$ (wavelength is from $1 \mathrm{~mm}$ to $30 \mu \mathrm{m}$ ) [14]. Thus, when the pixel size of such an SLM is close to the wavelength of $\mathrm{THz}$ radiation, the scattering and the diffraction effects are no longer negligible. In order to improve the overall signal to noise ratio, our new disk uses relatively larger circular holes of $2 \mathrm{~mm}$ diameter. Preliminary simulation and measurement results show that our new design effectively reduces the diffraction and the scattering effects caused by sharp corners of square holes.

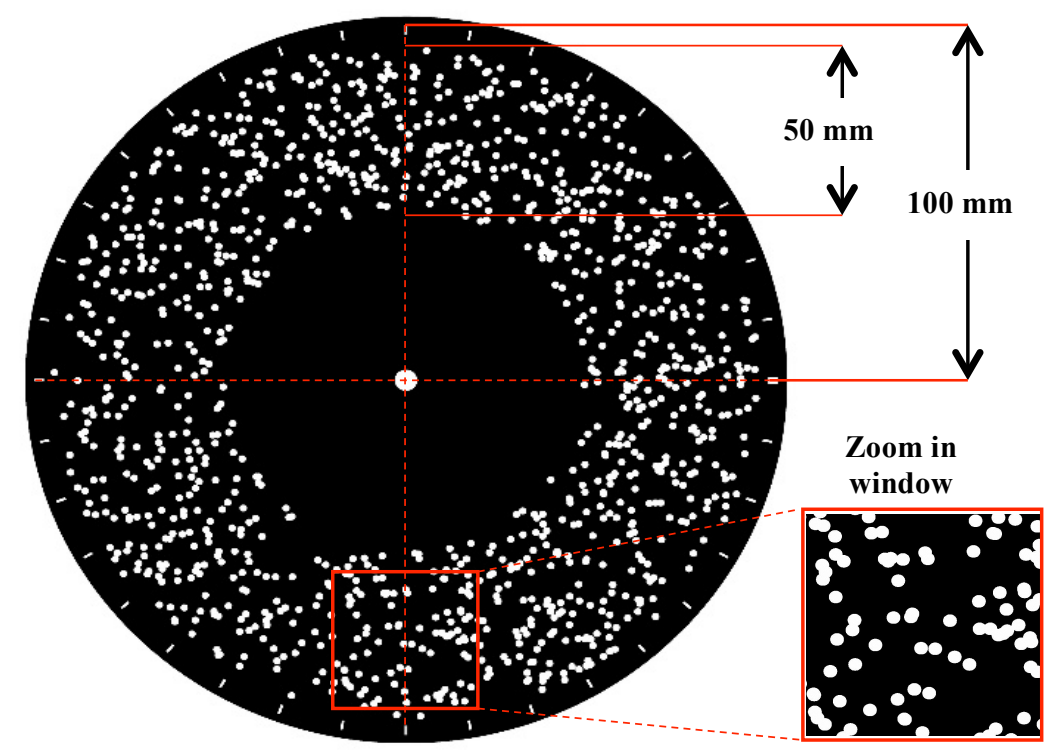

Fig. 1. Schematic diagram of the SLM spinning disk.

\subsection{Performances as Measurement Matrix}

Fig. 2 illustrates the partial measurement matrix generated from our SLM spin disk using 500 measurements. This matrix could be approximated as a block Toeplitz matrix. Haupt et al. [7] and Sebert et al. [15] have shown that Toeplitz-structured matrix is memory efficient with fast implementation. By making comparative analysis on different measurement matrices, we found that this disk shows good performances in image reconstruction. The corresponding results are shown in Fig. 3. In this test, the same parameters are set (including image size, sampling rate and reconstruction algorithm). More specifically, the image size is $64 \times 64$ pixels and each pixel represents $0.5 \mathrm{~mm} \times 0.5 \mathrm{~mm}$ sampling grid in practice. The size of each measurement matrix is $1200 \times 4096$ and each row corresponds to one measurement vector in practice. Thus the sampling rate is around $30 \%$, which is sufficient for obtaining high-quality 
results. The reconstruction algorithm used in this test is the minimum mean square error (MMSE) linear estimation [16].

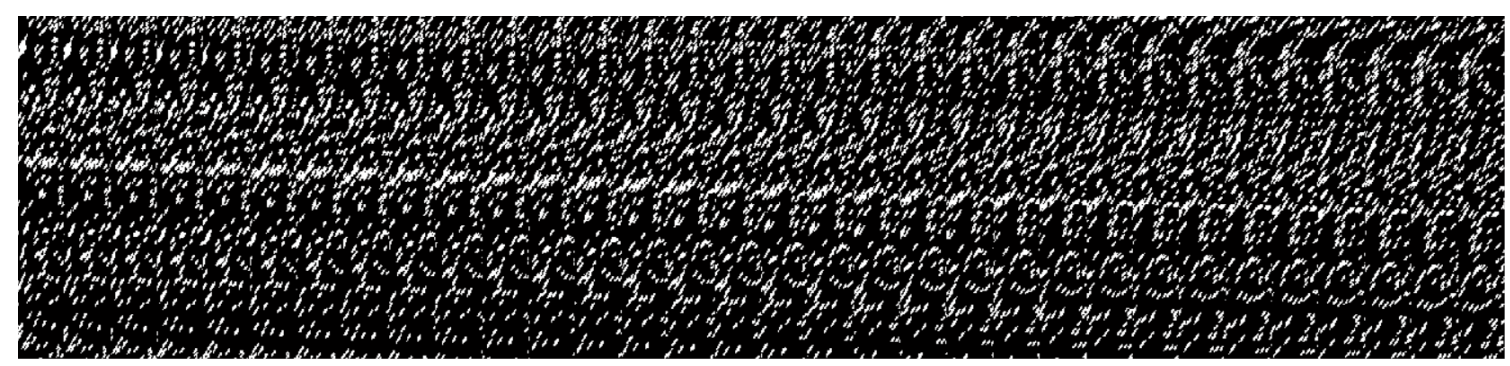

Fig. 2. Toeplitz-structured measurement matrix designed for our disk

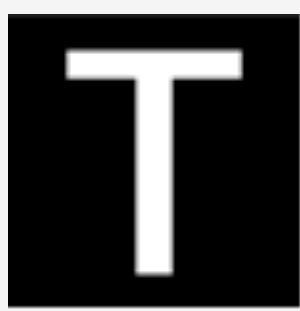

$a$

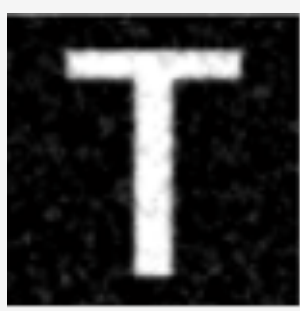

$b$

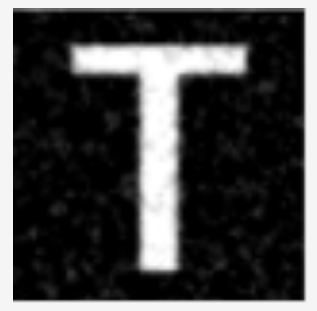

$c$

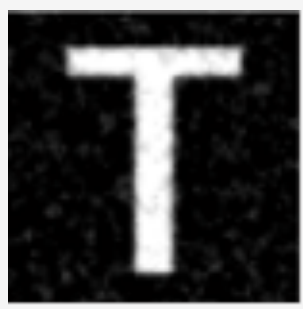

$d$

Fig. 3. Reconstruction performance test results

Subfigure a: Original image of letter ' $\mathrm{T}$ '

Subfigure b: Reconstructed image using random matrix

Subfigure c: Reconstructed image using partial Hadamard matrix

Subfigure d: Reconstructed image using the Toeplitz-structured matrix

Table 1 shows the comparison of the reconstructed images obtaining by using SLMs of a random matrix $(64 \times 64$ pixels, each pixel $0.5 \mathrm{~mm} \times 0.5 \mathrm{~mm})$, a partial Hadamard matrix $(64 \times 64$ pixels, each pixel $0.5 \mathrm{~mm} \times 0.5 \mathrm{~mm}$ ) and our spinning disk (2-mm-diameter circular holes). The evaluation parameters used are Peak Signal-to-Noise Ratio (PSNR), Structural Similarity (SSIM) index and Feature Similarity (FSIM) index [17 18]. Note that, the reconstruction quality achieved by using our measurement matrix (nearly Toeplitz-structured matrix) is close to that produced by full random CS measurement matrices and partial Hadamard matrix.

Table 1 Quality evaluation results

$\begin{array}{llll}\text { Measurement matrix } & \text { PSNR } & \text { SSIM } & \text { FSIM } \\ \text { Random matrix } & 19.15 & 0.93 & 0.56 \\ \text { Partial Hadamard matrix } & 19.12 & 0.94 & 0.56 \\ \text { Our spinning disk } & 19.00 & 0.93 & 0.57\end{array}$




\subsection{Spatial Resolution}

Based on our design, the similarity corresponding to two consecutive measurements with 0.5-degree disk rotation angle (720 measurements per circle) is approximate 0.5 (using correlation coefficient as assessment). Fig. 4 (a) and (b) shows the sampling performance of the mechanical SLMs based on square holes and circular holes respectively. To sense a linear-edge sample or an arc-edge sample, the minimum size of the sample that can be captured by using a square-holes-based SLM is limited by the square holes size. In contrast, the achievable sampling size by using a circular-holes-based SLM could be smaller than the diameter of such circular holes. As shown in Fig. 4, the diameter of the circular hole, $D$, is twice of the width of the square hole, $d$ (e.g., $D=2 d$ ). The minimum sampling size, $L$, is equal to the width of a square sampling hole. But the same dimension of $L$ can also be sampled by using a larger circular hole for both situations where the shape of the sample can be either straight (illustrated in Fig. 4 (a)) or arc (illustrated in Fig. 4 (b)).

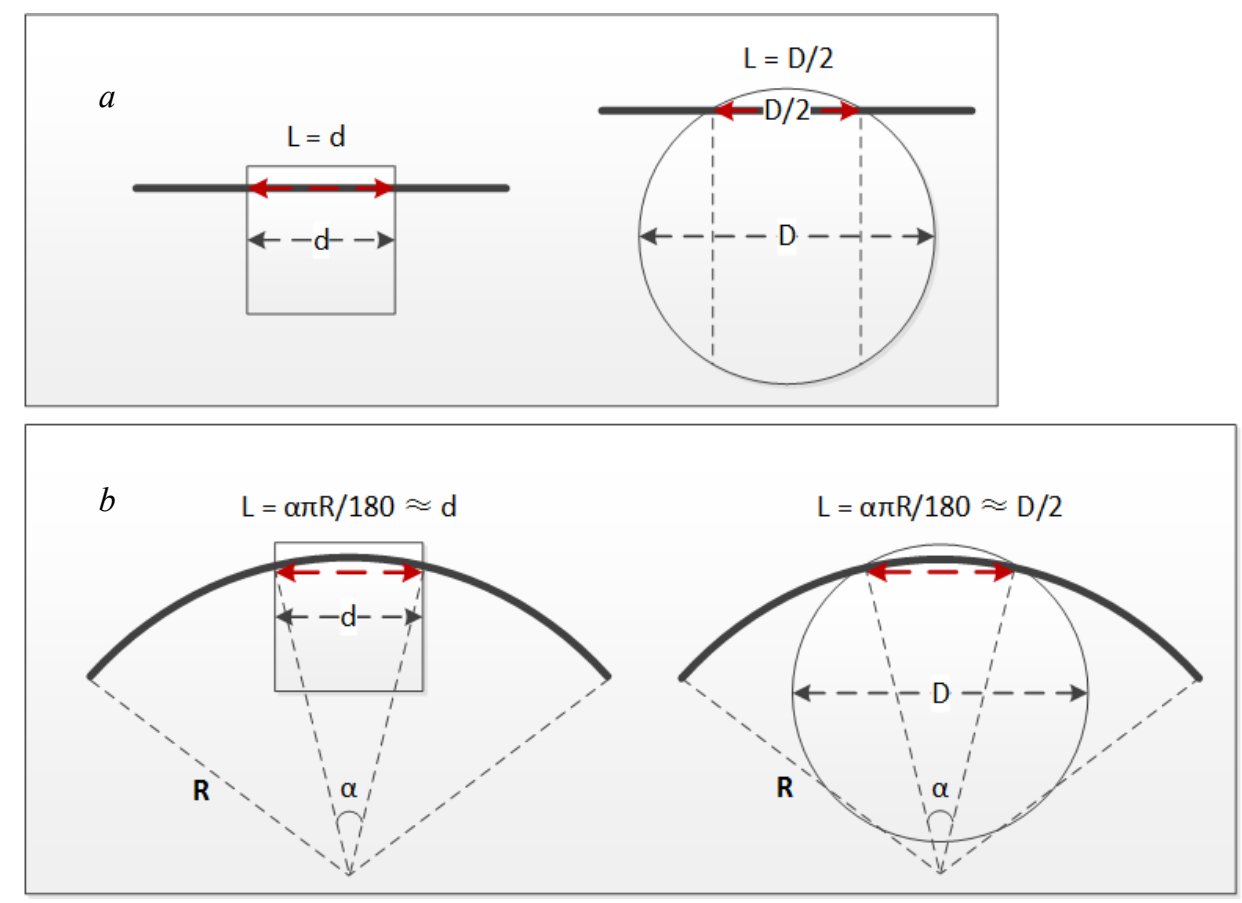

Fig. 4. Optimum sampling performance using square-hole-based SLM and circular-hole-based SLM Subfigure a: Sampling performance based on square holes Subfigure b: Sampling performance based on circular holes

In order to quantify the spatial resolution of our new spinning disk based compressed imaging system, we first studied a series of resolution test patterns through computer simulation. As shown in Fig. 5 (a), the widths of the white stripes of the test patterns are $6 \mathrm{~mm}, 3 \mathrm{~mm}$ and $2 \mathrm{~mm}$, respectively. In addition, a standard star pattern is also used for testing the spatial resolution. Fig. 5 (b) shows the reconstructed images of these test patterns, by using our 2-mm-diameter-hole SLM. Each image of $128 \times 128$ pixels was 
reconstructed using 2000 measurements, corresponding to a sampling rate of $12 \%$. A spatial resolution of better than $2 \mathrm{~mm}$ has been achieved with the 2-mm-diameter-hole SLM. This is significant particularly for THz imaging where the use of large holes is desirable since it allows $\mathrm{THz}$ radiation with longer wavelengths to pass through.

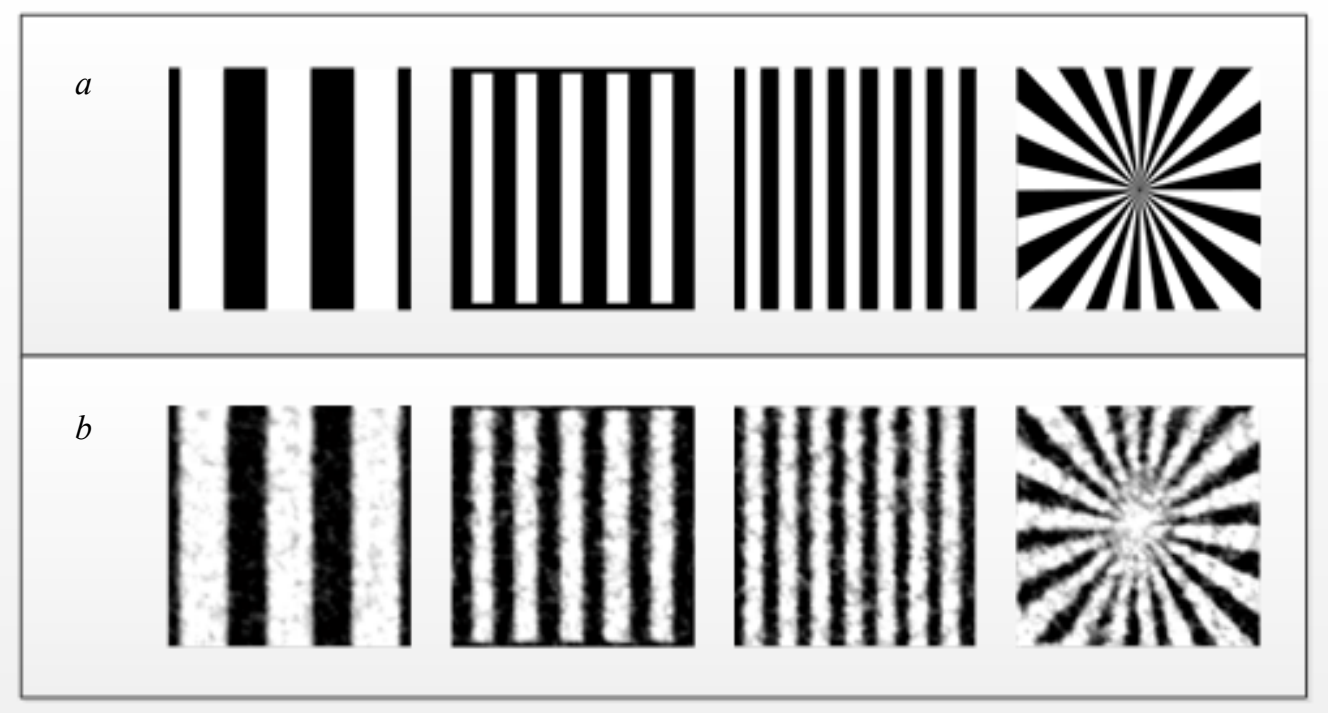

Fig. 5. Spatial resolution simulation test by using a series of resolution patterns ( $6 \mathrm{~mm}, 3 \mathrm{~mm}, 2 \mathrm{~mm}$, star pattern) Subfigure a: Original images of the resolution patterns

Subfigure b: Reconstruction images corresponding to a

\section{Experiment}

\subsection{Setup of Video Rate Compressed Imaging System}

Fig. 6 shows the schematic diagram of our video-rate compressed imaging system. The IR light from a source was collimated using a parabolic mirror. After transmitting through the sample and the SLM disk, the IR beam containing the optical information of the sample was then focused onto the detector by using another parabolic mirror. During imaging process, the SLM disk was rotated continuously and automatically, driven by an electric motor (Gearbox: PLG 42S, Motor: BG 40×25, Controller: BGE 40, Dunkermotoren). In order to synchronize the motor position with the data acquisition, a pair of laser diode and photodiode was used. The light beam from the laser diode propagates through the rectangle holes on the edge of the SLM disk which provides a precise mechanism for synchronization. In this system, we can obtain one image per each rotation thus the frame rate of the obtained video is determined by the rotation speed of the SLM disk. The rotation speed of the electric motor used in our system is 600 rotations per minute, providing a maximum image acquisition speed of about 10 frames per second. 
A motorized rotation stage (Thorlabs: CR1-Z7) was used to move a sample disk. 8 sample frames were fabricated on the sample disk and each frame has a size of $32 \mathrm{~mm} \times 32 \mathrm{~mm}$. As an example, Fig. 6 shows a ' $\mathrm{T}$ ' shape sample (the white represents $100 \%$ light transmission) which is fixed on one of the sample frames. Note that, this diagram only demonstrates the case where a rotation stage is used to change the samples. We could also use a motorized translation stage to change the samples.

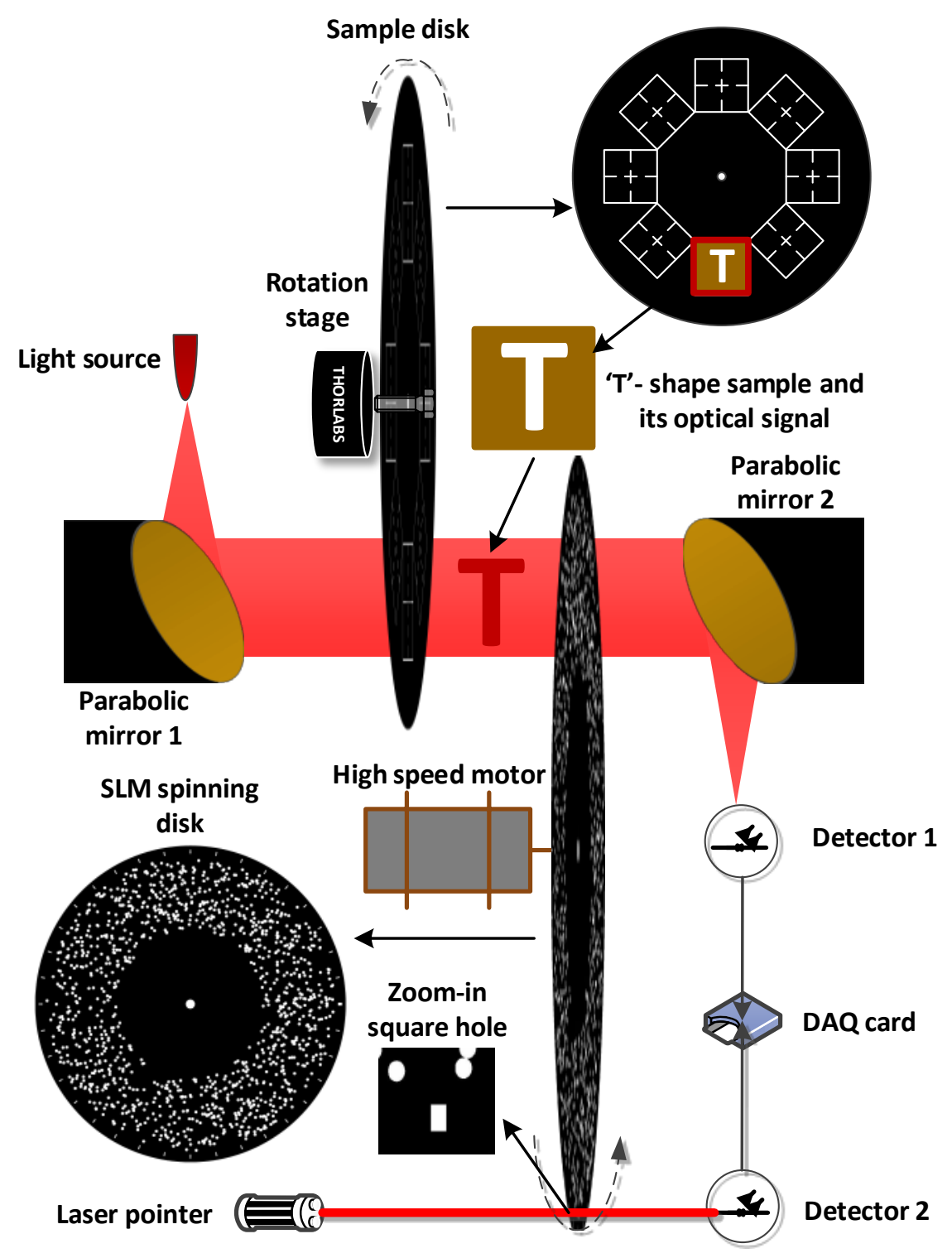

Fig. 6. Schematic diagram of the video rate compressed imaging system

\subsection{Reconstruction Strategy and Hardware Profile}

Unlike compressed imaging of single frame signal, the video rate compressed imaging increases the computational burden on reconstructing the image sequences. Considering this challenge, we have to design an efficient processing strategy. We firstly introduce the MMSE [16] as the reconstruction 
algorithm after balancing the reconstructed quality and time cost. This is subsequently combined with a paralleled processing approach to make full use of the multicore CPU. The computer we used for this imaging task is a quad-core CPU with 32 Gbyte RAM. The processing software is based on MATLAB 2015a and its corresponding toolboxes. The algorithms except the MMSE reconstruction mainly includes:

1) Gaussain pyramid for resampling the digital SLM spin disk; 2) Spline interpolation for obtaining the measurement signal from original sampling signal [19]; 3) Wiener filter for reducing the noise effect existed in reconstruction images [20].

Based on this processing strategy, 100 frame signals after captured by our compressed imaging system with a frame rate of $10 \mathrm{fps}$, can be reconstructed to100 images (each of $32 \times 32$ pixels) in 11.57 seconds, which corresponds to one image per every 0.12 seconds.

\section{Results and Discussions}

\subsection{Improvement of single-frame image quality}

In practical measurement there will be noise presented in the measured signal. Eq. 1 can be rewritten as $[21]$

$$
\boldsymbol{y}=\boldsymbol{\Phi} \boldsymbol{x}+\boldsymbol{\eta}
$$

where $\boldsymbol{\eta}$ represents the additive noise which may present in the measured signal. If the measured signal can be obtained for $K$ times $(K>1)$, then the average of $K$ measured signal $\overline{\boldsymbol{y}}$ is as

$$
\bar{y}=\frac{1}{K} \sum_{i=1}^{K}\left(\Phi x_{i}+\eta_{i}\right)=\Phi x+\frac{1}{K} \sum_{i=1}^{K} \eta_{i}
$$

where $i$ represents the $i$ th measurement and the expectation of $\overline{\boldsymbol{y}}$ is the measured signal without noises as

$$
E\{\overline{\boldsymbol{y}}\}=\boldsymbol{\Phi} \boldsymbol{x}
$$

Assuming that the noise presented in the measured signal is dominated by Gaussian additive white noise, the variance of the measured signal can be written as

$$
\sigma^{2} \bar{y}=\frac{1}{K} \sigma_{\eta}^{2}
$$

therefore, the standard deviation of $\overline{\boldsymbol{y}}$ is

$$
\sigma_{\bar{y}}=\frac{1}{\sqrt{K}} \sigma_{\eta}
$$

Eq. 7 and Eq. 8 show that the impact of noises can be lowered by increasing the number of measurements. Consequently, the average of the measured signal approaches to the original signal when 
increasing the number of measurements. Since our compressed imaging system can obtain large number of images at a video rate this provides an effective way to obtain high signal to noise ratio image for a static sample. Fig. 7 (a) shows the measured signals for a test sample which is made of $32 \mathrm{~mm} \times 32 \mathrm{~mm}$ copper tape with a 1-mm-wide cut-through stripe. The green solid line is the measured single-frame signal whilst blue and black solid lines are the averaged signal of 10-frame and 50-frame signals, respectively. In addition, the red dashed line represents the simulated signal. It is evident that averaging over multi-frame signal leads to improved signal to noise ratio (as illustrated in three zoom-in windows). Fig. 7 (b) shows the corresponding images reconstructed from these signals. Each image of $96 \times 96$ pixels was reconstructed from 1200 measurements (e.g., a sampling rate of 13\%). Again, the electric motor was rotating at its maximum speed of about 600 rotations per minute, corresponding to an image acquisition speed of 10 frames per second.

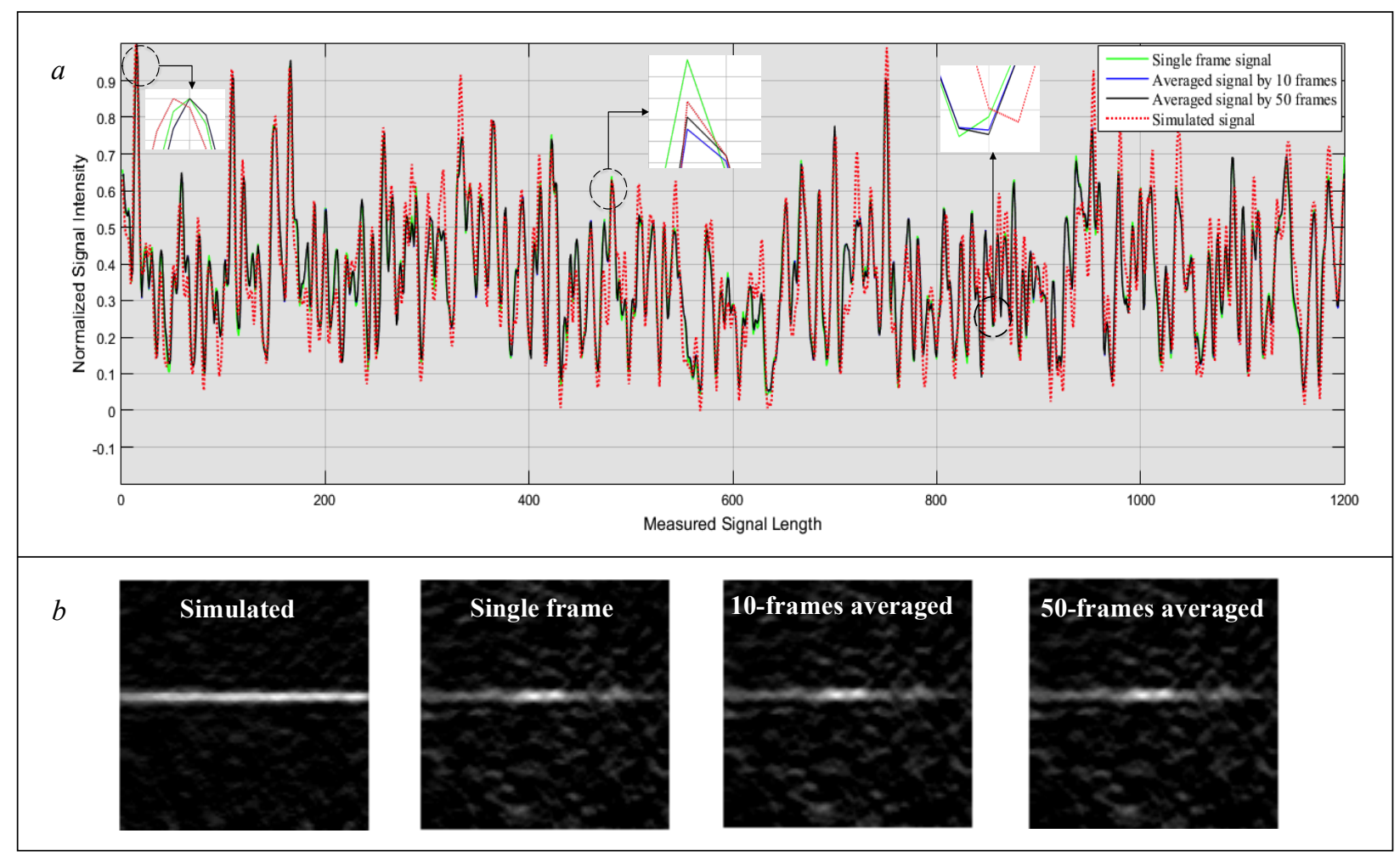

Fig. 7. Single-frame signal quality improvement by multi-frame signal average Subfigure a: Measured signals comparison

Subfigure b: Reconstructed images comparison corresponding to a

In order to quantify the spatial resolution, Fig. 7 (b) shows the reconstructed images using simulated and measured signals for the above-mentioned 1-mm-strip sample. Each image has $96 \times 96$ pixels covering an area of $32 \mathrm{~mm} \times 32 \mathrm{~mm}$, thus each pixel is $0.33 \mathrm{~mm} \times 0.33 \mathrm{~mm}$. The full width at half maximum (FWHM) of the 1-mm-wide-strip pattern, after taking into consideration of the line spread 
function (LSF), was found to be $1.0 \mathrm{~mm}$ and $1.1 \mathrm{~mm}$ for the reconstructed image using the simulated and measured signals, respectively (illustrated in Fig. 8). Therefore the achieved spatial resolution is $1.1 \mathrm{~mm}$ which is better than the hole diameter of $2 \mathrm{~mm}$.

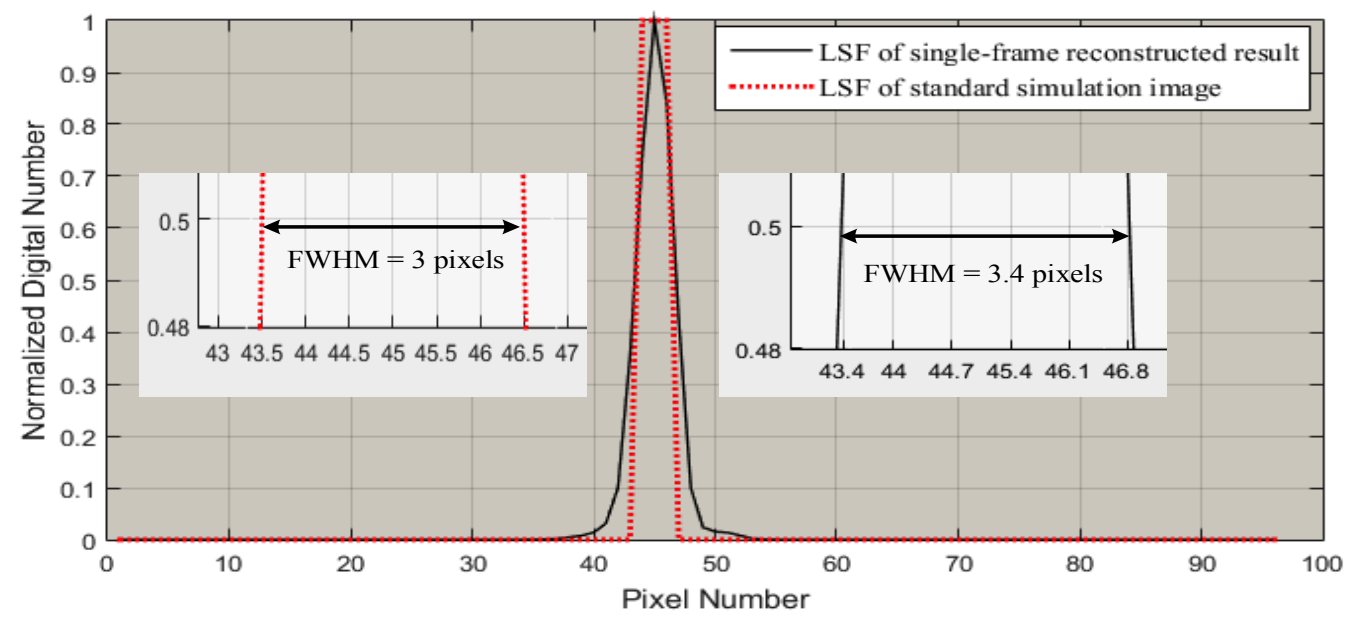

Fig. 8. LSF curve and FWHM calculation of the 1-mm stripe sample

\subsection{Video of moving samples}

Rapid imaging technology is highly desirable for time-critical application such as security check and inline inspection. In order to demonstrate its video rate imaging capability, we designed a test sample which is consisted of 8 alphanumeric and Chinese characters (e.g., 'A', 'M', '5', ‘7', ‘大', ‘化', ’中', ‘国'). The test sample is made of copper tape and the area occupied by the characters is $100 \%$ transparent to the light. The test samples were fixed on a sample disk which is mounted on a motorized rotation stage (Thorlabs: CR1-Z7). Each reconstructed images has $96 \times 96$ pixels and the image was reconstructed using 1200 measurements (e.g., 13\% sampling rate). When the sample '大' is moving across the sampling window, it can be imaged in real time. As an example, Fig. shows 15 images extracted from a video which is obtained at a frame rate of 9.85 fps. We can conclude that our compressed imaging system is capable of capturing video rate IR images. 
F. 166

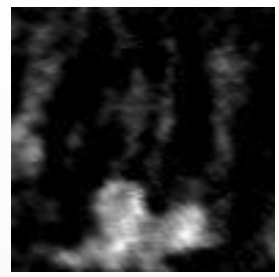

F. 171

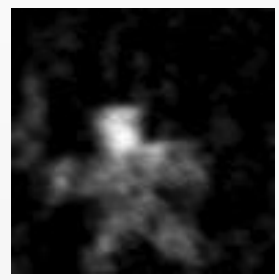

F. 176

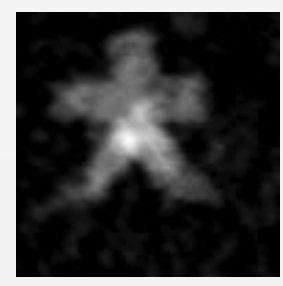

F. 167

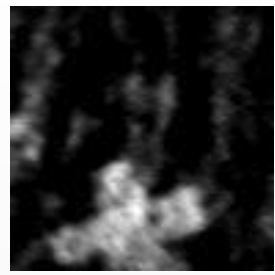

F. 172

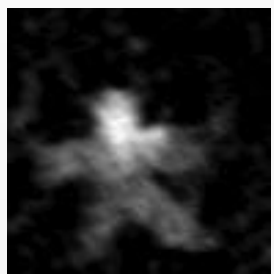

F. 177

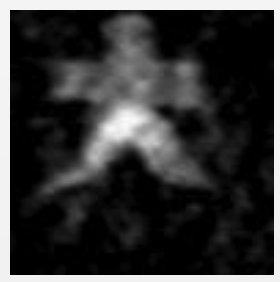

F. 168

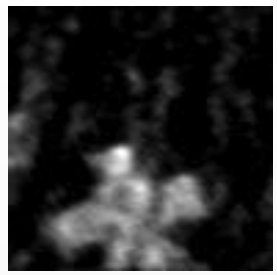

F. 173

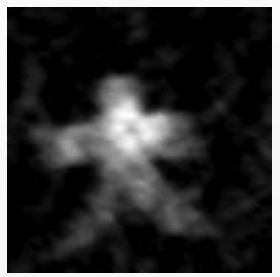

F. 178

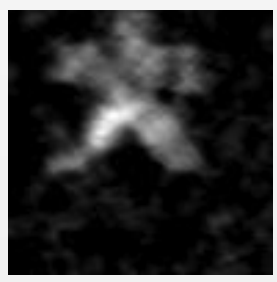

F. 169

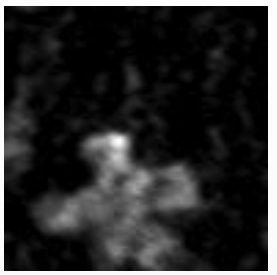

F. 174

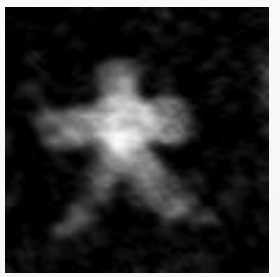

F. 179

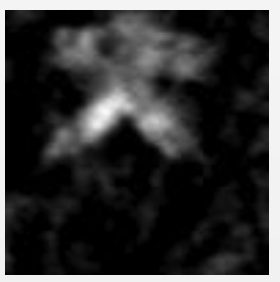

F. 170

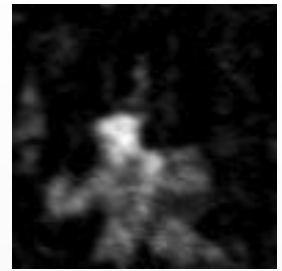

F. 175

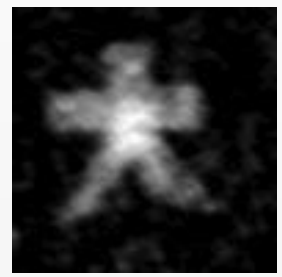

F. 180

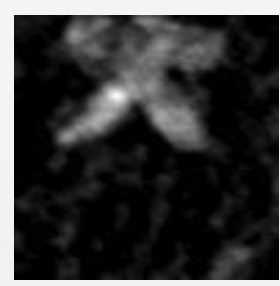

Fig. 9. Video rate compressed imaging for the moving sample '大'

\subsection{Imaging samples with large size}

An important application of IR and $\mathrm{THz}$ imaging is to detect the concealed objects. In many cases, the sample size might be larger than the field of view of the imaging system, thus it would be difficult to get the image of the whole sample. Here we will show that our video rate compressed imaging system, when combined with key-frame extraction technique [22], is capable of capturing the image of the whole sample even when the sample is larger than the imaging area of the imaging system.

As shown in Fig. 8 (a), the sample used is a 190-mm-long steel knife which is opaque to the light. The effective imaging area of our imaging system is $32 \mathrm{~mm} \times 32 \mathrm{~mm}$. The sample was fixed on a motorised translation stage (Physik Instrumente: M-521 Stage). The stage was moving at a speed of 6.6 $\mathrm{mm} / \mathrm{s}$. In total 301 images were acquired in 30 seconds (e.g., an imaging rate of about $10 \mathrm{fps}$ ). By dividing these images into a number of "shots" (49 frame images per shot) [23-25], we were able to automatically extract the key-frames. A complete knife image was finally generated by combining these key-frames (Fig. 8 (b)). Further signal processing can be used to enhance the quality and the visual effects of the image. Fig. 
8 (c) shows the image obtained by using a $5 \times 5$ median filter and grey scale inversion [26] whilst Fig. 8 (d) shows an image after edge sharpening using the Sobel operator [27].

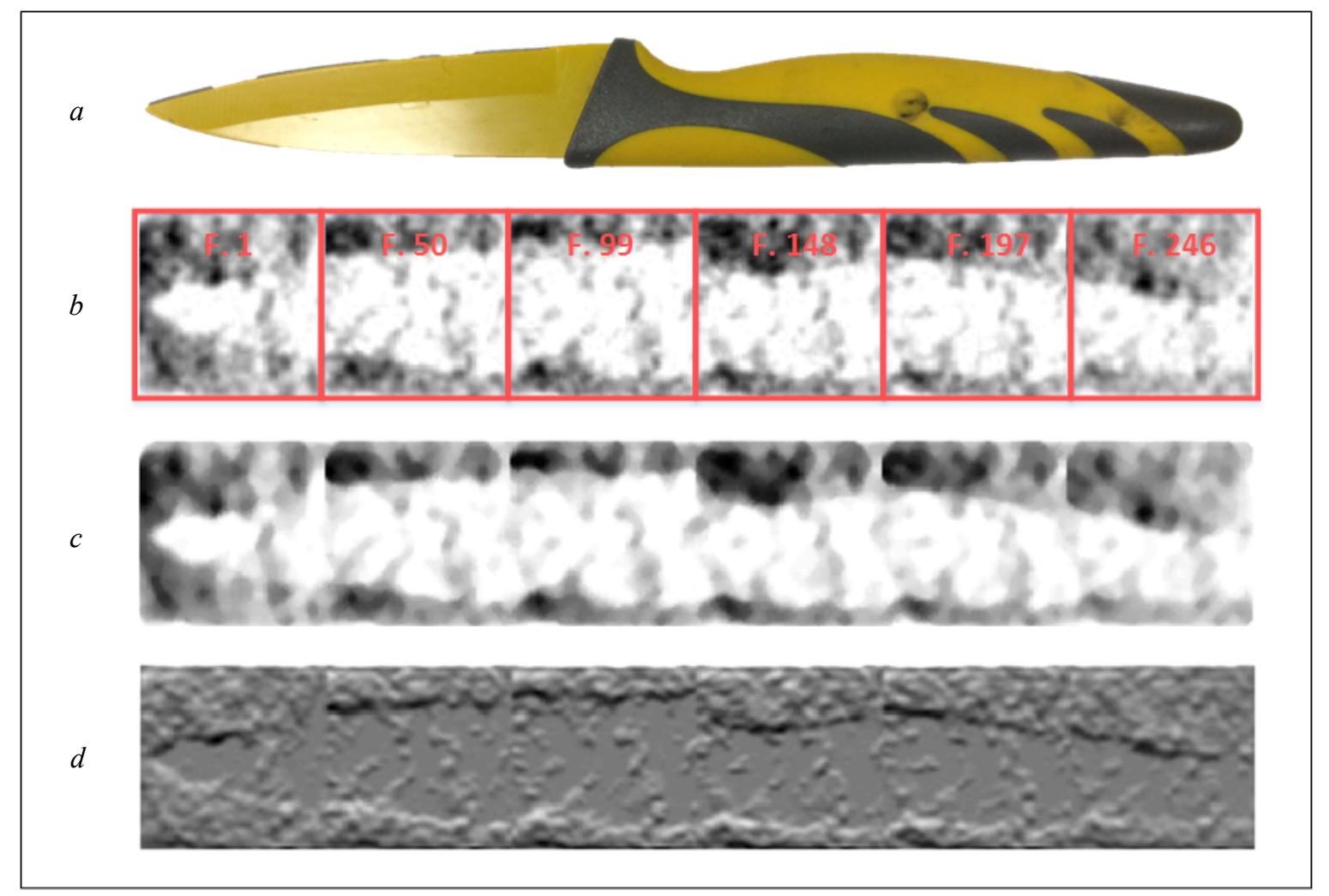

Fig. 8. Video rate compressed imaging test for a 190-mm steel knife (imaging region size $32 \mathrm{~mm} \times 32 \mathrm{~mm}$ ) Subfigure a: Steel knife sample picture

Subfigure b: Reconstructed images of the frame images of interest

Subfigure c: Processed mosaic image by using median filter and grey scale inversion

Subfigure d: Edge sharpening image for better recognition

\subsection{Terahertz Imaging Simulation}

As demonstrated above, we have achieved video rate imaging at IR frequencies using the proposed system based on the compressed sensing concept. Here we will perform further simulation to demonstrate that the same imaging system is applicable to $\mathrm{THz}$ imaging over a wide frequency range $(0.3 \mathrm{THz}-30$ $\mathrm{THz})$.

We firstly simulated optical transmission performances of the SLM with the increase of THz wavelength from $0.01 \mathrm{~mm}(30 \mathrm{THz})$ to $1 \mathrm{~mm}(0.3 \mathrm{THz})$. The upper left image in Fig. 11 (a) shows one of the original modulation areas on the SLM consist of many 2-mm-diameter circular sampling holes, where the holes are entirely transparent to the visible and IR light. However, when using the longer-wavelength $\mathrm{THz}$ radiation as the imaging beam, the transmitted power may be reduced due to the diffraction effects, particularly when the wavelength of $\mathrm{THz}$ radiation is close to the size of the 2-mm sampling holes [28]. 
Fig. 11 (a) shows the original transmission pattern of our disk-based SLM together with simulated ones calculated at $30 \mathrm{THz}, 3.0 \mathrm{THz}$, and $0.3 \mathrm{THz}$ respectively, whereas Fig. 11 (b) shows the diffraction pattern of $0.3-\mathrm{THz}$ radiation on the sample plane $(5-\mathrm{mm}$ distance to the SLM) after passing through one of 2-mm-diameter circular sampling holes. It is evident that $\mathrm{THz}$ radiation of $0.3 \mathrm{THz}$ (wavelength of $1.0 \mathrm{~mm}$ ) can still pass through the metal holes. As a further illustration, the image grid used in this simulation was $512 \times 512$ pixels covering an imaging area of $32 \mathrm{~mm} \times 32 \mathrm{~mm}$.

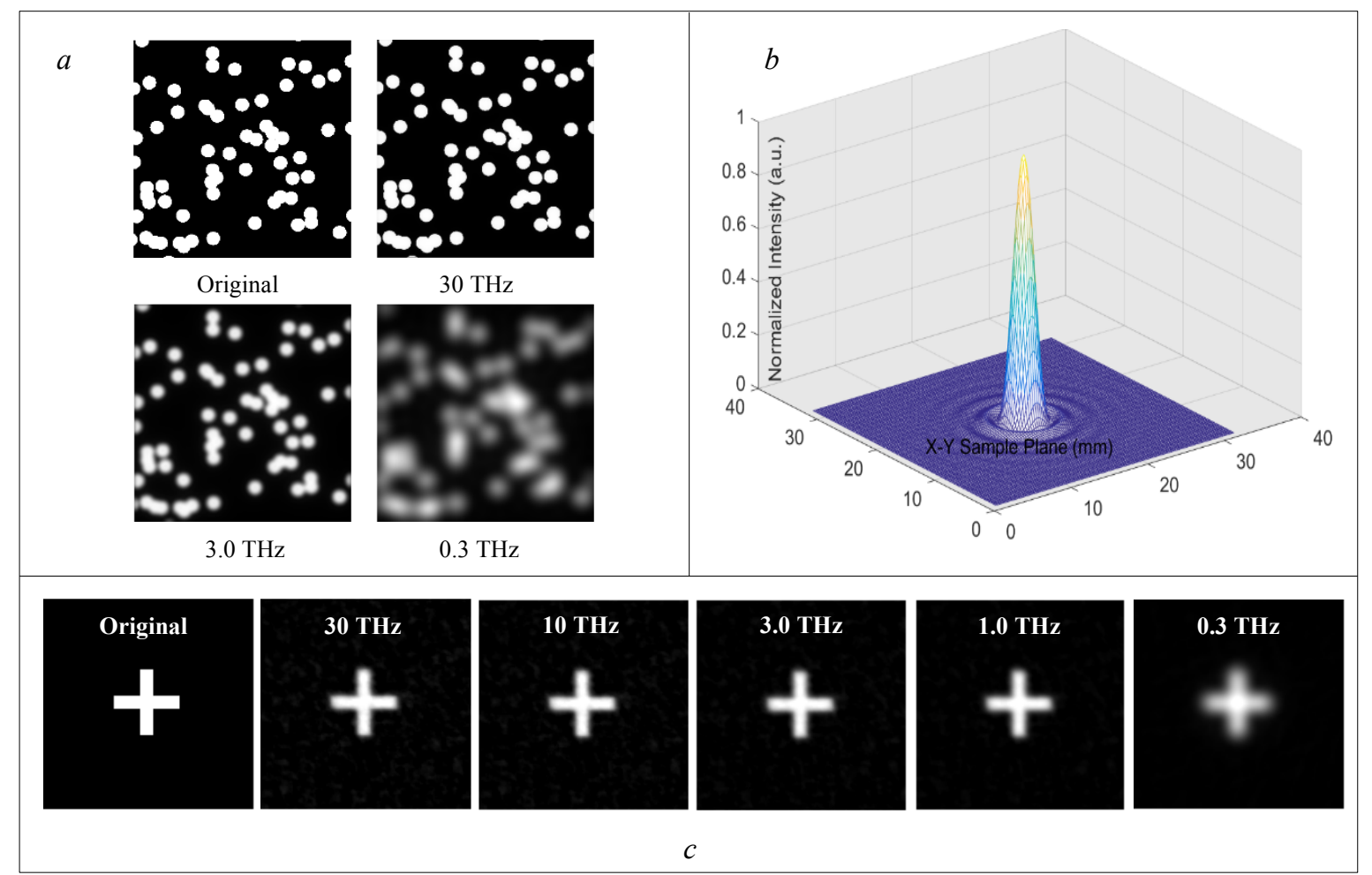

Fig. 11. THz imaging simulation

Subfigure a: Simulated SLM optical transmission performance at various $\mathrm{THz}$ frequencies

Subfigure b: Simulated diffraction effects of $1-\mathrm{mm}(0.3 \mathrm{THz}) \mathrm{THz}$ radiation

Subfigure c: Simulated compressed THz images in the frequency range of $0.3 \mathrm{THz}-30 \mathrm{THz}$

As further illustration, we simulated the compressed THz imaging using the optically degraded SLM (shown in Fig. 11 (a)). In the simulation, the sample used was a cruciform pattern as shown in the far left of Fig. 11 (c). The size of the original image is $128 \times 128$ pixels which is corresponding to $32 \mathrm{~mm} \times 32$ $\mathrm{mm}$ sampling area, and each stripe represents $2-\mathrm{mm}$ width (8 pixels) and 10-mm length (40 pixels). The other images in Fig. 11 (c), from left to right, are the reconstructed images simulated at a frequency of 30 $\mathrm{THz}, 10 \mathrm{THz}, 3.0 \mathrm{THz}, 1.0 \mathrm{THz}$ and $0.3 \mathrm{THz}$, respectively. We can find that the reconstructed cruciform samples become blurred with the increase in wavelengths. Nevertheless the pattern can still be recognized even at $0.3 \mathrm{THz}$ (wavelength of $1.0 \mathrm{~mm}$ ). We can therefore conclude that our novel SLM will be suitable 
for the $\mathrm{THz}$ compressed imaging from $0.3 \mathrm{THz}$ to $30 \mathrm{THz}$. In this simulation, the compression rate was set to $12 \%$ (2000 measurements) and the reconstructed algorithm was the MMSE [16].

\section{Conclusion}

In this paper, we have proposed a video rate imaging system based on the compressed sensing concept. Using a single pair of IR emitter and receiver, we demonstrate that the system is capable of capturing a $96 \times 96$ video sequence at 10 frames per second. The achieved spatial resolution is better than 2 $\mathrm{mm}$ using a novel SLM disk where the diameter of the cut-through hole is $2 \mathrm{~mm}$. In addition, our compressed imaging system with high imaging speed can be used to get high signal to noise image of a static sample via averaging over multi-frame signals and it can also be used to get a complete image of the whole sample even when the sample is larger than the field of view of the compressed imaging system. Furthermore, since our disk is made of stainless steel with chemically etched through-holes. The stainless steel is able to block both IR and $\mathrm{THz}$ radiations whilst the through-holes are transparent to all electromagnetic radiation. Given that the hole size is $2 \mathrm{~mm}$ in diameter and the wavelength of $\mathrm{THz}$ radiation is $0.3 \mathrm{~mm}$ at $1.0 \mathrm{THz}$, we anticipate that the proposed spin-disk approach will be applicable to compressed imaging at $\mathrm{THz}$ frequencies. The numerical simulations shows that the proposed spin-disk based SLM approach is suitable for compressed THz imaging covering a wide frequency range of $0.3-30$ $\mathrm{THz}$ which would be ideal for compressed $\mathrm{THz}$ time-domain system where the useful frequency region is usually $0.3-3.0 \mathrm{THz}$.

\section{Acknowledgments}

The work is partially supported by UK EPSRC (Research Grant EP/L019787/1 and EP/K023349/1).

\section{References}

[1] Donoho, D.L.: 'Compressed sensing', IEEE Trans. Inf. Theory, 2006, vol. 52, no. 4, pp. 1289-1306

[2] Candès, E.J., Tao, T.: 'Near optimal signal recovery from random projections: Universal encoding strategies?', IEEE Trans. Inf. Theory, 2006, vol. 52, no. 12, pp. 5406-5425

[3] Gehm, M.E., John, R., Brady, D.J., et al.: 'Single-shot compressive spectral imaging with a dual-disperser architecture', Opt. Express, 2007, vol. 15, no. 21, pp. 14013-14027

[4] Duarte, M.F., Davenport, M.A., Takhar, D., et al.: 'Single-pixel imaging via compressive sampling', IEEE Signal Processing Magazine, 2008, vol. 25, no. 2, pp. 83-91

[5] Candès, E.J., Romberg, J., Tao, T.: 'Stable signal recovery from incomplete and inaccurate measurements', Comm. Pure Appl. Math., 2006, vol. 59, no. 8, pp. 1207-1223 
[6] Candès, E.J., Tao, T.: 'Decoding by linear programming', IEEE Trans. Inf. Theory, 2005, vol. 51, no. 12, pp. 4203-4215

[7] Haupt, J.D., Bajwa, W.U., Raz, G.M., et al.: 'Toeplitz compressed sensing matrices with applications to sparse channel estimation', IEEE Trans. Inf. Theory, 2010, vol. 56, no. 11, pp. 5862-5875

[8] Rauhut, H.: 'Compressive sensing and structured random matrices', In: M, Fornasier (Ed.): 'Theoretical Foundations and Numerical Methods for Sparse Recovery' (De Gruyter, 2010), vol. 9, pp. 1-92

[9] Zhang, G., Jiao, S., Xu, X.: 'Compressed sensing and reconstruction with semi-hadamard matrices', in 2010 2nd International Conference on Signal Processing Systems (ICSPS), Dalian, China, 2010, vol. 1, pp. V1-194-V1-197

[10] Furxhi, O., Jacobs, E.L.: 'Image plane coded aperture detectors for THz imaging', in 2012 IEEE MTT-S International Conference on Microwave Symposium Digest (MTT), Montreal, QC, Canada, 2012, pp. 1-3

[11] Chan, W.L., Charan, K., Takhar, D., et al.: 'A single-pixel terahertz imaging system based on compressed sensing’, Applied Physics Letters, 2008, vol. 93, no.12, pp. 121105-1-121105-3

[12] Shen, H., Gan, L., Shen, Y.C., et al.: 'Spinning disk for compressive imaging', Optics letters, 2012, vol. 37, no.1, pp. $46-48$

[13] Vasile, T., Damian, V., Coltuc, D., et al.: 'Single pixel sensing for THz laser beam profiler based on Hadamard Transform', Optics \& Laser Technology, 2016, vol. 79, pp. 173-178

[14] Peiponen, K.E., Zeitler, A., Kuwata-Gonolami, M.: 'Generation and Detection of Terahertz Radiation'. In: W. T. Rhodes (Ed.): ‘Terahertz Spectroscopy and Imaging' (USA: Springer, 2013), vol. 171., pp. 1-25

[15] Sebert, F., Zou, Y.M., Ying, L.: 'Toeplitz block matrices in compressed sensing and their applications in imaging, in 5th International Conference on Information Technology and Application and Biomedicine, Shenzhen, China, 2008, pp. 47-50

[16] Gan, L.: 'Block compressed sensing of natural images', in 15th International Conference on Digital Signal Processing, Cardiff, UK, 2007, pp. 403-406

[17] Hore, A., Ziou, D.: 'Image quality metrics: PSNR vs. SSIM', in 20th International Conference on Pattern Recognition, Istanbul, Turkey, 2010, pp. 2366-2369

[18] Zhang, L., Zhang, L., Mou, X.Q., et al.: 'FSIM: a feature similarity index for image quality assessment', IEEE Trans. Image Processing, 2011, vol. 20, no. 8, pp. 2378-2386

[19] Hsieh, H., Andrews, H.: 'Cubic splines for image interpolation and digital filtering', IEEE Trans. Acoustics, Speech and Signal Processing, 1983, vol. 31, no. 4, pp. 532-540

[20] Islam, S.R., Maity, S.P., Ray, A.K.: 'On compressed sensing image reconstruction using multichannel fusion and adaptive filtering', in 2015 International Conference on Image Processing Theory, Tools and Applications (IPTA), Orleans, France, 2015, pp. 479-484

[21] Buades, A., Coll, B., Morel, J.M.: 'A review of image denoising algorithms, with a new one', Multiscale Modeling \& Simulation, 2006, vol. 4, no. 2, pp. 490-530 
[22] Calic, J., Izuierdo, E.: 'Efficient key-frame extraction and video analysis', in International Conference on Information Technology: Coding and Computing, 2002, pp. 28-33

[23] Zhuang, Y., Rui, Y., Huang, T.S., et al.: 'Adaptive key frame extraction using unsupervised clustering', in 1998 International Conference on Image Processing, Chicago, USA, 1998, pp. 866-870

[24] Ejaz, N., Tariq, T.B., Baik, S.W.: 'Adaptive key frame extraction for video summarization using an aggregation mechanism', Journal of Visual Communication and Image Representation, 2012, vol. 23, no. 7, pp. $1031-1040$

[25] Zhang, H.J., Wu, J., Zhong, D., et al.: 'An integrated system for content-based video retrieval and browsing', Pattern Recognition, 1997, vol. 30, no. 4, pp. 643-658

[26] Gallagher, N., Wise, G.: 'A theoretical analysis of the properties of median filters', IEEE Trans. Acoustics, Speech and Signal Processing, 1981, vol. 29, no. 6, pp. 1136-1141

[27] Gupta, S., Mazumdar, S.G.: 'Sobel edge detection algorithm', International journal of computer science and management Research, 2013, vol. 2, no. 2, pp. 1578-1583

[28] Li, Y.D., Li, Q., She, J.Y., et al.: 'Investigation on diffraction characteristics of continuous terahertz beam limited by hard-edge apertures', Optik-International Journal for Light and Electron Optics, 2013, vol. 124, no. 22, pp. 5669-5673 